\title{
Business model and methods of evaluation in sustainable manufacturing
}

\author{
Haishang $\mathrm{Wu}^{*}$ (D) \\ Faculty of Technology, University of Sunderland, Sunderland SR6 0DD, UK
}

Received: 25 August 2021 / Accepted: 13 October 2021

\begin{abstract}
Additive manufacturing (AM) enables cost-effective and efficient production toward sustainability. However, a rigorous evaluation method is required to further investigate the measurement method and efficiency before AM can be well-positioned in sustainable manufacturing and become the industry mainstream. Cost savings play a key role in the manufacturing industry. Compared to conventional manufacturing (CM), the cost of AM is volume-independent. In contrast, CM production requires a certain volume to share the initial tooling costs to achieve cost reduction. This constraint limits CM from service on demand and leaves ambiguity in the threshold setting of that critical batch volume. In addition, the invisibility of AM advantages in cost factors blocks AM technologies from appropriate processes and affects its applications. To address these issues, this paper proposes a business model. The major issues encountered by AM are the scaling, speed, and size of products. The enhancement of cost modeling and addressing speed, scale, and size issues are the novelties of this study and provide a breakthrough in AM issues. Generic equations are derived using the convergence effect and cost-volume intersection calculation between AM and CM. Furthermore, the divide-and-conquer approach is proposed to support scaling factors and dependencies for both AM and CM. Consequently, appropriate AM technologies can be compared with the CM convergence threshold to contribute to decision-making. Next, the advantages and weaknesses of AM are identified, and a collaboration pattern is proposed to connect large enterprises, small-and medium-sized enterprises, and home-based manufacturers into an AM society. Through this society, the advantages of AM can be fully exploited, scaling and speed issues can be addressed, and AM's dominant role in sustainable manufacturing can be made feasible.
\end{abstract}

Keywords: sustainability / material recycling / collaboration / localization / standard / AM / CM / SME

\section{Introduction}

Industrial ecology encourages the formation of synergies between companies across various industrial sectors [1] to systematically review the waste seen as an abundant, local, and free resource [2]. Therefore, increasing manufacturing efficiency through process and recycling competence is the key to sustainable plastic manufacturing [3]. Recycling and reduction of material waste are both clear advantages of most AM technologies [4]. In this study, the advantages of material recycling and starting with AM are completely exploited, and best-fit technologies and products are recommended.

This paper begins with an evaluation method for additive manufacturing (AM) and conventional manufacturing $(\mathrm{CM})$ processes. In addition, the measurement approach and collaborative pattern are explored.

\footnotetext{
* e-mail: haishang.wu@research.sunderland.ac.uk
}

This paper aims at sustainable recycling and manufacturing and proposes a framework that covers the collectionrecycling-manufacturing (CRM) model, business model, and strategy control model. Among these, the CRM model deals with technical aspects; the strategy control model guides human factors; and the business model, which is the focus of this study, aggregates the assessment results of the CRM model into a benchmarking to form a cost model. This study focuses on the AM scaling issue and industrial collaboration, which are tactical factors that need to be resolved.

Before proceeding to the main body of this study, the following flowchart (Fig. 1) provides an overview of the methodologies and patterns, as well as the evaluation method and approach used to illustrate the business model context. The business model is introduced to elaborate on the following sections: Strategy, Methodologies, Approaches, Discussion, Challenges, and Conclusion. 


\begin{tabular}{|c|c|c|c|c|c|c|c|c|c|c|}
\hline Section 1 & Section 2 & Section 3.1 & Section 3.2 & Section 4 & & Section 5.1 & Section 5.2 & Section 5.3 & Section 6 & Section 7 \\
\hline Introduction & Method & \multicolumn{3}{|c|}{ Approaches and Results } & & \multicolumn{3}{|c|}{ Discussion } & \multicolumn{2}{|c|}{ Conclusion } \\
\hline $\begin{array}{c}\text { Issue } \\
\text { Identification }\end{array}$ & $\begin{array}{l}\text { Method of } \\
\text { Evaluation }\end{array}$ & $\begin{array}{c}\text { Conceptual } \\
\text { Cost Modeling }\end{array}$ & $\begin{array}{c}\text { CM/AM Cos } \\
\text { Modeling }\end{array}$ & st Results & $\checkmark$ & $\begin{array}{c}\text { Cost Modeling } \\
\text { Capability }\end{array}$ & Uses Colla & $\begin{array}{l}\text { akness and } \\
\text { orative pattern }\end{array}$ & Challenges & Conclusion \\
\hline
\end{tabular}

Fig. 1. Flowchart of business model context, methodologies, and evaluation criteria.

Section 2, Methodologies: Sustainable manufacturing aims to reduce costs, material waste, energy consumption, and $\mathrm{CO}_{2}$ emission. However, due to the lack of industry standards and measurement methods, quantitative analysis can be difficult. This paper proposes a business model to envision the methodologies of the cost model and the collaborative pattern.

Sections 3 and 4, Approaches and Results: The cost model proposal starts with a conceptual design and ends with a review of the results. From the supply chain and logistics viewpoints, the cost model mainly covers the upstream quantitative analysis, and the collaborative pattern mainly covers the downstream qualitative analysis.

The "divide-and-conquer" method divides and calculates the total cost of CM into initial tooling cost and unit cost and derives the unit cost of different batch volumes and the convergence of CM cost. The qualitative analysis of cost saving and transportation reduction illustrates that a standardized collaboration through large enterprises (LEs), small-and medium-sized enterprises (SMEs), and home-based manufacturers (HBMs) can help local recycling and manufacturing, as well as distributed supply, to further reduce the cost and transportation of AM.

Section 5, Discussion: The capability and uses of cost modeling patterns, as well as the weaknesses and limitations of the cost model, are discussed. Different technologies of AM [stereolithography (SLA) and fusion deposition modeling (FDM)] are benchmarked against CM in terms of different batch volumes to produce a decision support evaluation. In addition to the cost model, the collaborative pattern is elaborated as an enhancement to support the business model in terms of supply chain management, logistics, and localization.

Sections 6 and 7, Challenges and Conclusion: This paper further identifies the challenges, and then presents the conclusion. Challenges are the issues of AM currently facing, which include a precise measurement and benchmarking that this study attempts to resolve, and the associated resolutions on the concerns regarding sizing, scaling, and speed issues.

\section{Methods}

AM has been widely applied to highly complex products, particularly complicated shapes or colors, which are differentiators of CM [5]. AM can flexibly achieve a part change without impacting other parts. AM is being increasingly used in the medical, automotive, and aerospace industries, particularly in lightweight parts for energy-saving and part replacement for different types of products [6].
In Section 3, the methods of AM and CM cost modeling are proposed, where CM applies different volumes $(20,000$ and 100,000) and AM applies different technologies (SLA and FDM) to demonstrate individual impact factors in producing generic formulas without any testing data. In Section 4 , live data are used to test the formula under predefined conditions and derive generic equations. These equations illustrate the quantitative analysis and can be generically applied to any computation with input data ready to produce prediction; they can be developed into a decision support software package.

\subsection{Issue identification}

AM has some disadvantages that must be resolved before mass production. Neither AM nor CM has established a concrete cost model to quantify individual unit costs and initial costs. The invisibility of cost estimation between $\mathrm{AM}$ and $\mathrm{CM}$ has been a bottleneck to decision support. Furthermore, compared to AM, CM is fast in the fabrication of parts, while AM prints products at lower speed and is limited to producing premium quality, smaller quantity, and smaller size [7]. With the advancement of technologies, such disadvantages are resolvable and shall not affect AM's role at all. However, the cost model is crucial to benchmarking for decision support, which is the objective of this study. AM's versatile technologies favor cost reduction through the generic formula applied in this paper. Furthermore, AM can fully utilize the proposed collaborative pattern in a full-scale provision [8]. It supports the cost model and offers a good opportunity for HBMs to solve scale and speed issues through its easy entry and inexpensive initial costs.

\subsection{Methods of evaluation}

An extensive investigation reveals the feasibility of seamless integration of material recycling with AM. AM is the optimal choice for rapid prototyping, as it offers the flexibility to make necessary changes in a most rapid and cost-effective manner. It uses a computer-aided design (CAD) file and a converted stereolithography (STL) file to calculate the triangles and sliced shape of each layer to be printed $[9,10]$. Through the additive layer-by-layer method, AM achieves a significant reduction in design effort and requires much less materials than $\mathrm{CM}$ to create a product prototype once the CAD and STL software are ready $[11,12]$. Compared to CM, which is a subtractive technology, AM achieves significant waste reduction by consuming only the exact materials the products require [13]. This reduces energy consumption as well as $\mathrm{CO}_{2}$ 
Table 1. Cost modeling format for benchmarking.

\begin{tabular}{llllll}
\hline Item/Method & Denotation & AM_method_A & AM_method_B & CM_20k & CM_100k \\
\hline Labor & & $L$ & $L$ & & \\
Assembly & $' \mathbf{O}$ ' & $\boldsymbol{V H}$ & $\boldsymbol{H}$ & $L$ & $L$ \\
Materials & & 0 & 0 & $\boldsymbol{V H}$ & $H$ \\
Machine & $' \mathbf{T}$ & $M$ & $H$ & $M$ \\
Tooling & 'C' & $\mathrm{C}_{\mathrm{AM}}$ & $\mathrm{C}_{\mathrm{AM}}$ & $\mathrm{C}_{\mathrm{CM}(20 \mathrm{k})}$ & $\mathrm{C}_{\mathrm{CM}(100 \mathrm{k})}$ \\
& Total & any & & 20,000 & 100,000 \\
Batch volume & 'n' & & & & \\
\hline
\end{tabular}

VH: very high; H: high; M: medium; L: low.

emission. In addition, AM can produce a part that requires the assembly of many parts from CM and contributes to energy saving through a better material yield.

Cost modeling differs for AM and CM. The unit cost of $\mathrm{AM}$ is constant, as it does not require any tooling; however, CM requires a higher batch volume to achieve cost reduction due to the initial tooling cost, which is the dominant cost [14]. By taking advantage of such unique characteristics of AM, this study investigates the point of intersection at which $\mathrm{CM}$ gains scaling advantage over $\mathrm{AM}$. It further computes the CM convergence, in which an alternative AM technology can offer a lower cost and gain cost advantages over the cost of CM convergence.

\section{Approach}

The benchmarking approach is critical in cost reduction and decision support [15]. The cost of AM is volumeindependent, while $\mathrm{CM}$ production requires a higher volume to achieve cost reduction. This constraint limits CM from service on demand and leaves ambiguity in the threshold setting of that critical batch volume.

A rigorous approach is proposed to analyze individual cost items and their dependencies in the aerospace industry. The divide-and-conquer [16] approach develops a cost-modeling pattern in AM and $\mathrm{CM}$ evaluation. Finally, general equations are derived through the intersection of $\mathrm{AM}$ and $\mathrm{CM}$ and the convergence of $\mathrm{CM}$ to address the scaling issue. In parallel, the BTF ratio [17] in a relevant area is investigated to evaluate the input weight over the output weight. Ti-64 is the material used in benchmarking to support BTF in cost modeling.

\subsection{Generic formula for cost modeling}

In cost modeling, two AM methods (method A and method B) and two CM volume sizes (20k and 100k) are illustrated in the format indicated in Table 1 to measure the interferences of volume and technologies against cost. The setup cost of tooling is " 0 " in the AM case, which means that AM does not require tooling. However, this cost is very high in the CM case, which needs to be broken down into each part of the unit. The convergence effect is applied to investigate the lowest net cost of CM. It produces a generic formula without any input data, but once the input data are ready, it can be applied to any condition to produce generic equations for benchmarking.

Through cost patterns, this study develops a generic business model formula that can be used in AM and CM cost modeling. The formulas are variable-dependent, and the generic form can be developed into a software package for decision support. There are two generic formulas. The first one is "divide," which breaks the tooling cost down through volume dependency, and the second one is "conquer," which aggregates the results into a total cost calculation.

\section{$3.2 \mathrm{CM}$ and $\mathrm{AM}$ cost modeling}

\section{Generic form of tooling cost (mold design and mold} cost) for CM

Tooling unit cost $\times$ Part counts $=$ Mold design cost + mold unit cost $\times$ Part counts

\section{Generic form of total cost for CM}

Total unit cost $\times$ Part counts $=$ Mold design cost $+($ mold unit cost + Other unit cost $) \times$ Part counts

The parameters used to translate the generic formulas into parametric formulas are listed in Table 2.

CM tooling for "Divide": Tooling $\left(\mathrm{T}_{\mathrm{cm}}\right)$ in formula 1 is divided into " $\mathrm{M}$ " and " $\mathrm{m}$ " and translated into:

$$
\mathbf{T}_{\mathrm{CM}} \times \mathbf{n}=\mathbf{M}+\mathbf{2 0 0 0 0} \mathbf{m}
$$

The balance equation can be represented by applying two individual scales to derive " $\mathrm{M}$ " and " $\mathrm{m}$ ":

$$
\begin{gathered}
\mathrm{T}_{\mathrm{CM}(20 \mathrm{k})} \times 20000=\mathrm{M}+20000 \times \mathrm{m}(\text { for CM_20 } \mathrm{k}) \\
\mathrm{T}_{\mathrm{CM}(100 \mathrm{k})} \times 100000=\mathrm{M}+100000 \times \mathrm{m}(\text { for CM_100 } \mathrm{k})
\end{gathered}
$$

Through the two equations:

$$
\begin{aligned}
\mathbf{M} & =\mathbf{2 5 0 0 0} \times\left(\mathbf{T}_{\mathbf{C M}(20 \mathrm{k})}-\mathbf{T}_{\mathbf{C M}(100 \mathrm{k})}\right) \\
\mathbf{m} & =1.25 \times \mathbf{T}_{\mathbf{C M}(100 \mathrm{k})}-0.25 \times \mathbf{T}_{\mathbf{C M}(20 \mathrm{k})}
\end{aligned}
$$


Table 2. Parameters used in cost modeling.

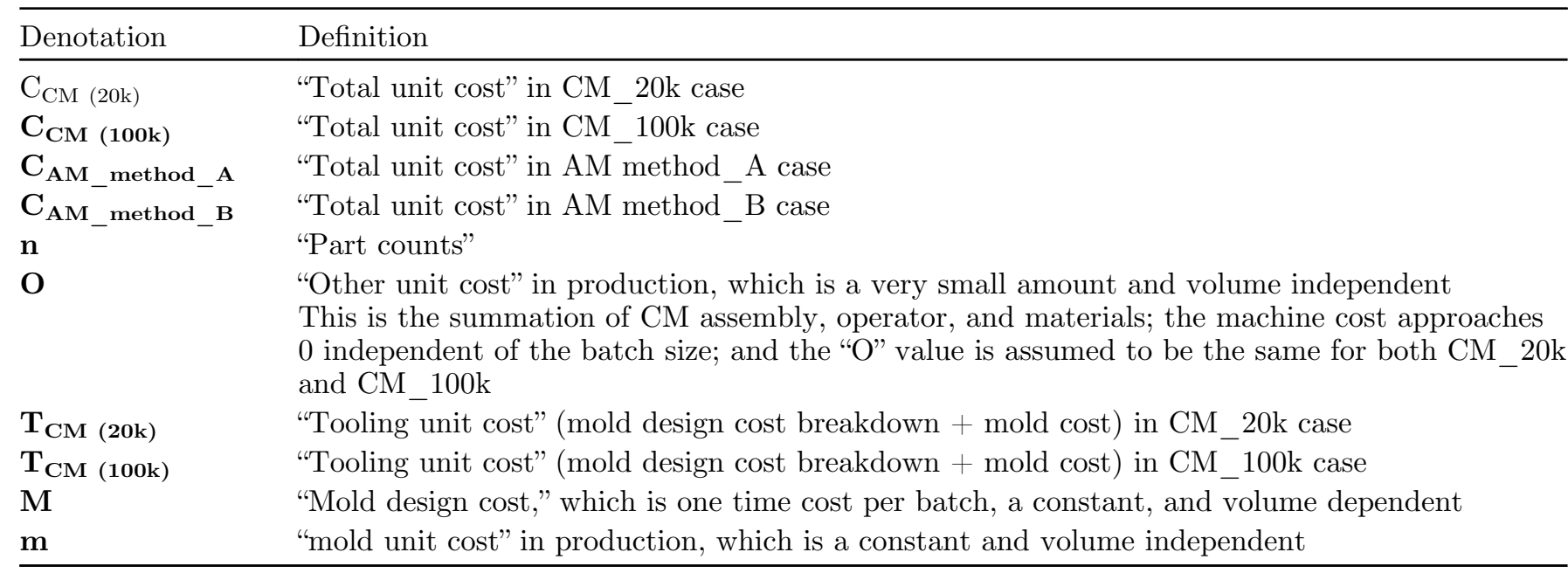

CM cost of modeling for "Conquer": By taking the values "M" (molding design cost) and "m" (mold unit cost) from formula 1 into a total cost calculation;

$$
\begin{gathered}
\mathrm{C}_{\mathrm{CM}(20 \mathrm{k})} \times 20000=\mathrm{M}+(\mathrm{m}+\mathrm{O}) \times 20000\left(\text { for } \mathrm{CM}_{20 \mathrm{k}}\right) \\
\mathrm{C}_{\mathrm{CM}(100 \mathrm{k})} \times 100000=\mathrm{M}+(\mathrm{m}+\mathrm{O}) \times 100000\left(\text { for } \mathrm{CM}_{100 \mathrm{k}}\right)
\end{gathered}
$$

Under any condition, the total cost in a generic form can be represented as; $\mathrm{C}_{\mathrm{CM}} \times \mathrm{n}=\mathrm{M}+(\mathrm{m}+\mathrm{O}) \times \mathrm{n}$

$$
\text { which derives } \mathbf{C}_{\mathrm{CM}}=\frac{\mathbf{M}}{\mathbf{n}}+(\mathbf{m}+\mathbf{O})
$$

The CM generic formula can be applied to any scenario, including the two CM individual cases, which implies the following:

With a given "T," we can derive "M" and "m" values.

With a given "O" and the derived "M" and "m" values, each $\mathrm{C}_{\mathrm{CM}}$ value can be derived with a given " $\mathrm{n}$ " value.

This also implies that the generic formula enables CM unit cost estimation $\left(\mathrm{C}_{\mathrm{CM}}\right)$ under any volume size "n."

\section{AM cost modeling}

In contrast, "C $\mathrm{C}_{\mathrm{AM}}$," the unit cost value in the AM case, stays constant and is volume-independent.

$$
\mathbf{C}_{\mathrm{AM}}=\mathbf{C}_{\text {constant }}
$$

\section{Results}

As indicated in Figure 2, AM and CM cost benchmarking has become feasible for investigating the concerns in terms of

- The expected volume at which CM starts to gain a cost advantage over AM, which refers to $\left(n_{1}, c_{1}\right)$ in the AM$\mathrm{CM}$ intersection.

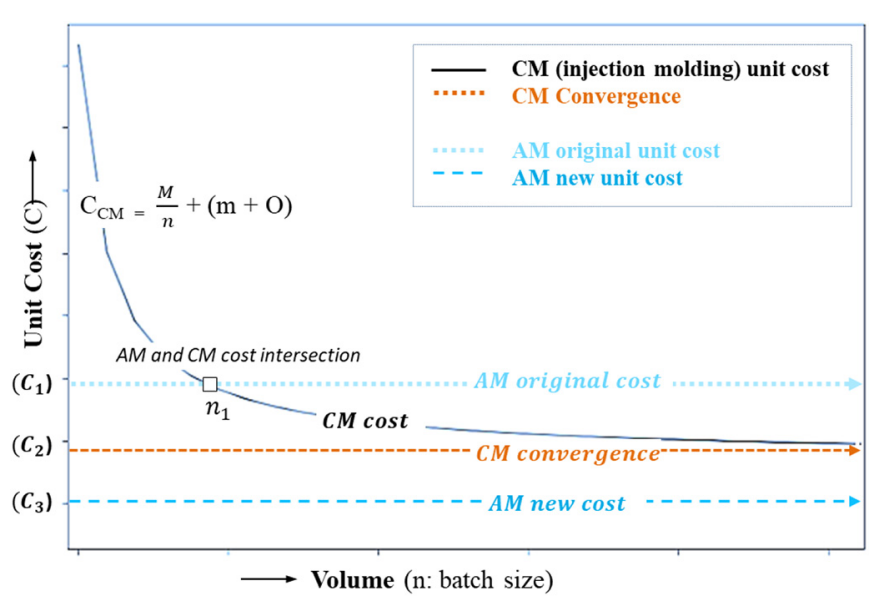

Fig. 2. Cost-volume correlation in cost modeling.

- The CM convergence in which CM reaches the lowest cost, regardless of the volume, which refers to the $\left(\infty, c_{2}\right)$ of the red dashed line.

- The expected AM technology that reduces cost to an even lower value than that of CM convergence $\left(c_{2}\right)$, to gain cost advantage over CM. This refers to $c_{3}-$ a shift from the red dashed line to the blue line based on AM technology.

Figure 2 Cost-volume correlation in cost modeling

The investigation results indicate the following three concerns and provide a solution based on equations (1)-(4):

1. To estimate the $\mathrm{CM}$ volume at which the $\mathrm{CM}$ cost starts to win over the $\mathrm{AM}$ cost, let $\mathrm{C}_{\mathrm{CM}}=\mathrm{C}_{\mathrm{AM}}=\mathrm{C}_{1}$ at the intersection. This derives $\mathbf{C}_{\mathbf{C M}}=\frac{\mathrm{M}}{\mathrm{n}}+(\mathrm{m}+\mathrm{O})=\mathbf{C}_{\mathbf{A M}}=$ $\mathrm{C} 1$ or $\mathrm{M}+(\mathrm{m}+\mathrm{O}) \times \mathrm{n}=\mathbf{C}_{\mathbf{A M}} \times$ nat the $\mathbf{C}_{\mathbf{C M}} / \mathbf{C}_{\mathbf{A M}}$ intersection.

With the derived "M" and "m" values and the given "O" and " $\mathrm{C}_{\mathrm{AM}}$ " values, intersection occurs at $\left(\mathrm{n}_{1}, \mathrm{c}_{1}\right)$; the "n" (volume) value at $\mathrm{C}_{\mathrm{CM}}$ and $\mathrm{C}_{\mathrm{AM}}$ intersection can be easily derived by subtracting the AM unit cost from the unit cost of molding and other cost of CM divided by 
the mold design cost:

$$
\mathbf{n}_{\text {interaction }}=\frac{\mathbf{C}_{\mathrm{AM}}-(\mathbf{m}+\mathbf{O})}{\mathbf{M}}
$$

In other words, when $n \geq n_{\text {intersection }}$, the CM volume starts to gain a cost advantage over AM.

2. To find the convergence of $\mathrm{CM}$ cost at $\mathrm{C}_{\text {convergence }}\left(\mathrm{C}_{2}\right)$, the discrete notation can be expressed as follows:

Let c convergence be the CM cost at convergence:

$\forall(\mathrm{c}, \mathrm{n}) \in\left\{\left(\mathrm{c}_{0}, \mathrm{n}_{0}\right),\left(\mathrm{c}_{1}, \mathrm{n}_{1}\right) \ldots\left(\mathrm{c}_{\infty}, \mathrm{n}_{\infty}\right)\right\}, \exists \mathrm{c}_{\text {convergence }}$ satisfies $c_{\text {convergence }} \leq c_{n} \forall c_{n} \in\left\{c_{0}, c_{0} \ldots c_{\infty}\right\}$ The generic equation $\mathrm{C}_{\mathrm{CM}}=\frac{\mathrm{M}}{n}+(\mathrm{m}+\mathrm{O})$ can be expressed by $\mathbf{C}_{\mathbf{A} M} \times \mathbf{n}-\mathbf{M}-(\mathbf{m}+\mathbf{O}) \times \mathbf{n}=0$

Let $\left(\mathrm{n}, \mathrm{C}_{\mathrm{CM}}\right)$ be mapped to the $(\mathrm{x}, \mathrm{y})$ Cartesian coordinate system:

$$
\begin{aligned}
& \mathbf{C}_{\mathrm{CM}} \times \mathrm{n}-\mathrm{M}-(\mathrm{m}+\mathrm{O}) \times \mathrm{n}=0 \text { is converted into } \mathrm{yx} \\
& -\mathrm{M}-(\mathrm{m}+\mathrm{O}) \times \mathbf{x}=0
\end{aligned}
$$

The deviation of the $\mathrm{y}$-axis $\left(\mathrm{C}_{\mathrm{CM}}\right)$ against the $\mathrm{x}$-axis $(\mathrm{n})$ becoming 0 at convergence indicates;

$$
\begin{aligned}
& \frac{\partial(\mathrm{yx}-\mathrm{M}-(\mathrm{m}+\mathrm{O}) \times \mathbf{x})}{\partial \mathrm{x}}=0, \\
& \text { which derives : } \mathbf{y}_{\text {convergence }}-0-(\mathrm{m}+\mathrm{O})=0 \\
& \text { This implies } \mathbf{C}_{\text {convergence }}=(\mathbf{m}+\mathbf{O})
\end{aligned}
$$

Equation (6) is the solution of the $2^{\text {nd }}$ investigation, which fully explains CM convergence calculation.

3. When the $\mathrm{AM}$ unit cost drops from $\mathbf{C}_{\mathrm{AM} \_ \text {method_A }}$ to $\mathbf{C}_{\mathrm{AM} \text { _method_B }}\left(\mathrm{C}_{3}\right)$, which is equal to or lower than $\mathrm{C}_{\mathrm{CM} \text {-convergence, }}$ no matter how large the $\mathrm{CM}$ volume is, $\mathrm{C}_{\mathrm{CM}}$ will not be able to achieve $\mathbf{C}_{\mathrm{AM} \_ \text {method_B }}$, which is the new AM cost.

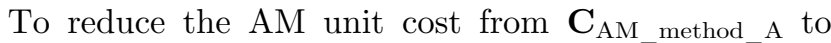
$\mathbf{C}_{\mathrm{AM} \text { _method_B }} \leq \mathrm{C}_{\mathrm{CM} \text { (convergence) }}$, the AM method needs to be adjusted.

As machines and materials contribute to the majority of AM's costs, both are the best candidates for cost reduction.

\section{AM method for cost reduction}

As $\mathrm{CM}$ convergence occurs at $\mathbf{C}_{\mathbf{C M}}$ (convergence) $=$ $(\mathrm{m}+\mathrm{o})$, the convergence value can be lower than that observed in method A. However, with the new AM method

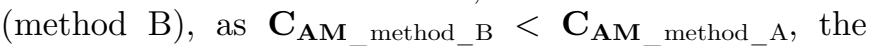
unit cost of $\mathrm{AM}$ lower than that of $\mathrm{CM}$ is feasible. As indicated in Table 3, the new cost format is: $\mathbf{C}_{\mathbf{A M} \text { _method_B }}$ $<\mathbf{C}_{\mathbf{C M} \text { (convergence) }}$

$$
\text { whichimplies } \mathbf{C}_{\mathrm{AMmethodB}}<(\mathbf{m}+\mathbf{O})
$$

The investigation yields the following results:

- CM batch size at a value of $n=\frac{\mathrm{C}_{\mathrm{AM}}-(\mathrm{m}+\mathrm{O})}{\mathrm{M}}$ or higher will gain a cost advantage over AM.

- $\mathrm{CM}$ cost convergence occurs at $\mathrm{C}_{\mathrm{CM} \text { (connvergence) }}=(\mathbf{m}+$ $\mathbf{O}$ ), where $\mathbf{C}_{\mathbf{C M} \text { (convergence) has the lowest cost. }}$

- When the AM method is changed with lower machine or materials costs, the new $\mathrm{AM}$ cost, $\mathbf{C}_{\mathrm{AM} \text { new }}$, might
Table 3. AM materials and machine cost format.

\begin{tabular}{lll}
\hline Method & AM_ method_A & AM_method_B \\
\hline Materials & $V H$ & $H$ \\
Machine & $V H$ & $H$ \\
\hline
\end{tabular}

VH: very high; H: high.

become lower than $\mathbf{C}_{\mathrm{CM}}$ (convergence). An example is illustrated in the next section using live data.

\section{Discussion}

\subsection{Cost modeling capability}

The cost modeling concept is introduced in section 3, where live data are tested against the generic formulas for verification. Table 4 illustrates the different focuses under $\mathrm{AM}$ and $\mathrm{CM}$ benchmarking conditions, which test different AM technologies and different batch sizes for $\mathrm{CM}$. The test data are fed into the formula and generic equations and results are derived based on the predefined conditions, which can be easily applied to different onsite cases.

\section{Input data and predefined conditions}

In this cost benchmarking example, the application is in the aerospace industry. Injection molding is the technology used in CM, where the batch sizes for benchmarking are $20 \mathrm{k}(20,000)$ and $100 \mathrm{k}(100,000)$, respectively. SLA is the original technology used in AM (AM_method_A), and FDM is the new AM technology (AM method B). Among all AM technologies, FDM extrusion is one of the most cost-effective and easy to prepare [18]. Hence, it is selected to demonstrate a new cost benchmark (AM_method B). Plastic is the material used in the evaluation, and raw data obtained from Deloitte Insight [19] are used to demonstrate cost modeling for how to derive generic equations to contribute to decision support.

AM costs depend on the technology being used and is volume-independent. In contract, CM cost is volumedependent, so different batch volumes of CM are applied in benchmarking.

As indicated in Table 5, for the 20,000 pieces of CM parts, the unit cost (\$3.61) is 2.5 times higher than that of AM (\$1.41); however, when the batch size is increased to 100,000 pieces, the unit price drops to $\$ 1.39$, which is slightly lower than AM's cost. Among the different cost items, tooling (including molding) dominates and contributes to over $95 \%$ of CM's cost [20].

\section{Derivation of generic equations}

The AM cost pattern is different from the $\mathrm{CM}$ cost pattern, as machines and materials contribute $60 \%$ and $30 \%$ of the AM cost, respectively [21]. By taking the "divide-and-conquer" approach, derived from generic formulas, into the parametric calculation, we can obtain the following: 
Table 4. Cost modeling benchmarking conditions.

\begin{tabular}{lllll}
\hline Conditions & AM_method_A & AM_method_B & CM_20k & CM_100k \\
\hline Method & Stereolithography (SLA) & Fusion deposition modeling (FDM) & Injection molding & Injection molding \\
Batch size & any & any & $\mathbf{2 0 , 0 0 0}$ & $\mathbf{1 0 0 , 0 0 0}$ \\
Materials & Plastic & Plastic & Plastic & Plastic \\
\hline
\end{tabular}

Table 5. Live data for AM and CM benchmarking.

\begin{tabular}{llll}
\hline Method & AM_SLA & CM_20k & CM_100k \\
\hline Assembly & 0.01 & 0.06 & 0.05 \\
Operator & 0.14 & 0.01 & 0.01 \\
Materials & 0.43 & 0.01 & 0.01 \\
Machine & 0.83 & 0.05 & 0.02 \\
Tooling & 0 & 3.48 & 1.30 \\
Total & $\$ \mathbf{1 . 4 1}$ & $\mathbf{\$ 3 . 6 1}$ & $\mathbf{\$ 1 . 3 9}$ \\
\hline
\end{tabular}

(\$/part) (raw data: Deloitte insight).

\section{CM tooling cost per batch and per unit}

Formula (2) indicates $\mathrm{M}=25000 \times\left(\mathrm{T}_{\mathrm{CM}} \quad\right.$ (20k) $\left.\mathrm{T}_{\mathrm{CM}}(100 \mathrm{k})\right) \mathrm{m}=1.25 \times \mathrm{T}_{\mathrm{CM}(100 \mathrm{k})}-0.25 \times \mathrm{T}_{\mathrm{CM}(20 \mathrm{k})} \mathrm{By}$ applying the data in Table 5,

$$
\begin{aligned}
\mathbf{M} & =25000 \times(3.48-1.30)=54,500 \mathbf{m} \\
& =1.25 \times 1.30-0.25 \times 3.48=0.755
\end{aligned}
$$

This derives $\mathrm{M}=54,500 \mathrm{~m}=0.755$, which means that the mold design costs $\$ 54,500$ per batch, and the mold costs $\$ 0.755$ per unit.

\section{CM total cost per unit}

The summation of the four items, assembly, operator, materials, and machine cost, account for approximately 0.1 and only $5 \%$ of the total cost, which is independent of the batch size. To simplify the equation, let the sum of these four items be the "other cost": "o"= $\mathbf{0 . 1}$, which is a fixed cost independent of batch size.

Formula (3) indicates: $\mathrm{C}_{\mathrm{CM}}=\frac{\mathrm{M}}{n}+(\mathrm{m}+\mathrm{O})$

By applying the data in Table 5 ,

$$
\mathrm{C}_{\mathrm{CM}}=\frac{54500}{n}+(0.755+0.1) \quad \mathrm{CCM}=\frac{54500}{\mathrm{n}}+0.855
$$

\section{AM cost modeling}

In contrast, the " $\mathrm{C}_{\mathrm{AM}}$ " value in the $\mathrm{AM}$ case remains constant (\$1.41) and volume-independent.

$$
\begin{aligned}
& \text { Formula (4) indicates CAM } \\
& =\mathbf{C} \text { constant By applying the data in Table 5, } \\
& \quad \mathbf{C A M}=1.41
\end{aligned}
$$

\section{Key parameter calculation}

1. To estimate the $\mathrm{CM}$ volume at which the $\mathrm{CM}$ cost starts to win over the $\mathrm{AM}$ cost, let $\mathrm{C}_{\mathrm{AM}}=\mathbf{C}_{\mathrm{CM}}$ be the equilibrium point. This yields $1.41=\frac{54500}{n}+0.855$ at equilibrium, and the "n" value can be easily derived as $\frac{54500}{n}=0.555$

In other words, when $\mathbf{n} \geq \mathbf{9 8 1 9 8}$, the CM volume starts to gain cost advantage over the AM volume.

2. To find the convergence of CM cost at $c_{\infty}$, the discrete notation can be expressed as follows:

Let $\mathrm{c}_{\text {convergence }}$ be the $\mathrm{CM}$ cost at convergence.

The generic equation $\mathrm{C}_{\mathrm{CM}}=\frac{54500}{5}+0.855$ can be expressed as $\mathbf{C}_{\mathrm{CM}} \times \mathrm{n}-54500-0.855 \times \mathrm{n}=0$

Let $\left(\mathbf{C}_{\mathrm{CM}}, \mathrm{n}\right)$ be mapped to the $(\mathrm{y}, \mathrm{x})$ Cartesian coordinate system.

The deviation of the y-axis (" $\mathbf{C}_{\mathrm{CM}}$ ") against the $\mathrm{x}$-axis ("n") becomes 0 at convergence, which leads to $\frac{\partial(\mathrm{xy}-54500-0.855 \mathrm{x})}{\partial \mathrm{x}}=0$, which indicates $\mathrm{y}_{\text {convergence - }}$ $0-0.855=0$ or $\mathbf{y}$ convergence $=\mathbf{0 . 8 5 5}$ This implies that the CM unit cost becomes flat when approaching $\mathrm{CM}$ convergence, $\mathbf{\$ 0 . 8 5 5}$ is the lowest unit cost, despite the batch size.

3. When the AM unit cost drops from $\$ 1.41$ to $\mathrm{CM}$ convergence or below the convergence value $\left(\mathrm{C}_{\mathrm{AM}} \leq\right.$ 0.855 ), irrespective of how large the $\mathrm{CM}$ volume is, $\mathrm{CM}$ cannot achieve this AM costs. To reduce the AM unit cost from $\$ 1.41$ to $\$ 0.855$, or even lower, the AM method needs an adjustment. In the Deloitte case, as machines and materials contribute $60 \%$ and $30 \%$ of AM's cost, respectively, both cost values are targets for cost reduction.

\section{AM method for cost reduction}

Among all AM technologies, FDM extrusion is one of the most cost-effective and easy-to-prepare options. Hence, it is selected to demonstrate a new cost benchmark. According to the AM machine and material benchmark [22], the material and machine costs of AM differ for different methods. 
Table 6. Material and machine costs of AM for different methods.

\begin{tabular}{lll}
\hline Method & AM_SLA & AM_FDM_Extrusion \\
\hline Materials & $\$ 175-250 / \mathrm{kg}$ & $\$ 20 / \mathrm{kg}$ \\
Machine & $\$ 200,000+$ & $\$ 150,000$ \\
\hline
\end{tabular}

(data source: Simpson, T).

Table 7. Full scale of AM and CM cost items.

\begin{tabular}{lllll}
\hline Method & AM_SLA & AM_FDM_Extrusion & CM_20k & CM_100k \\
\hline Assembly & 0.01 & 0.01 & 0.06 & 0.05 \\
Operator & 0.14 & 0.14 & 0.01 & 0.01 \\
Materials & 0.43 & 0.04 & 0.01 & 0.01 \\
Machine & 0.83 & 0.62 & 0.05 & 0.02 \\
Tooling & 0 & 0 & 3.48 & 1.30 \\
Total & $\mathbf{\$ 1 . 4 1}$ & $\mathbf{\$ 0 . 8 1}$ & $\mathbf{\$ 3 . 6 1}$ & $\mathbf{\$ 1 . 3 9}$ \\
\hline
\end{tabular}

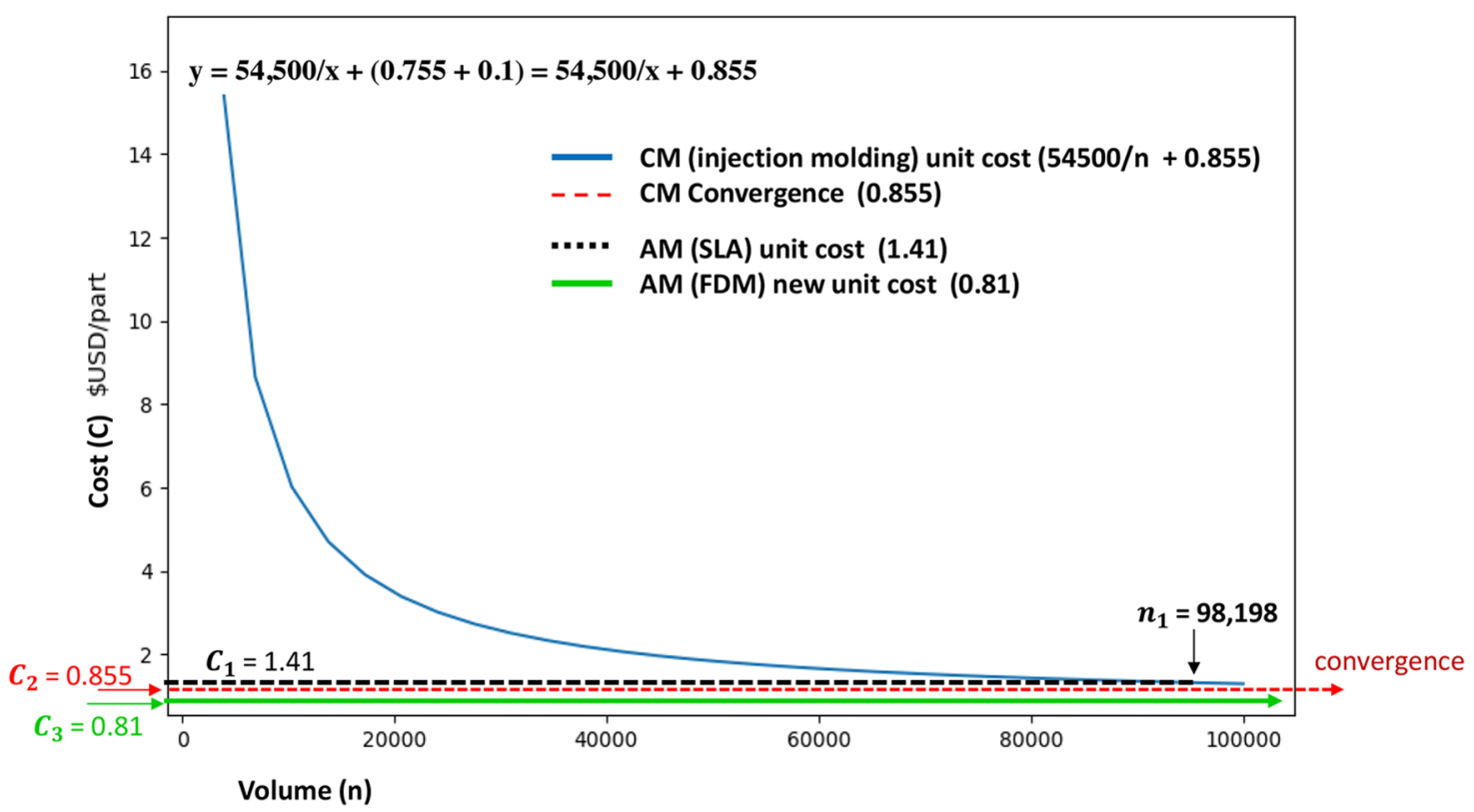

Fig. 3. Convergence benchmark of $\mathrm{CM}$ and $\mathrm{AM}$ costs based on three investigations.

Table 6 indicates that the estimation of material comparison between SLA and FDM is approximately 1.0:0.1 and the machine cost is approximately 1.0:0.75; hence, both values $(0.1$ and 0.75$)$ are applied in a conversion from SLA cost to FDM cost.

In addition to machines and materials, other costs can also be similar for both SLA and FDM. The details of the full-scale cost items of the two AM methods and two CM methods are listed in Table 7 .

Figure 3 shows the comparison between AM (both FDM and SLA technologies) and CM (both 20k and 100k) by substituting the data obtained from Table 7 into a plotting. The Figure further demonstrates the results obtained from the live case, as indicated in the following three key statements:

At $\mathrm{C}_{1} \quad\left(\mathbf{C}_{\mathrm{AM}}(\mathrm{SLA})=1.41\right)$, the intersection occurs at $\mathrm{n}_{1}=98198$. When $\mathrm{n}<\mathrm{n}_{1}, \mathbf{C}_{\mathrm{AM}}<\mathbf{C}_{\mathrm{CM}}$ and vice versa.

At $\mathrm{C}_{2}\left(\mathbf{C}_{\mathrm{CM}}\right.$ (convergence) $\left.=0.855\right), \mathbf{C}_{\mathrm{CM}}$ approaches the lowest cost value when the batch size is increased.

At $\mathrm{C}_{3}\left(\mathbf{C}_{\mathrm{AM}(\mathrm{FDM})}=0.81\right), \mathbf{C}_{\mathrm{AM}}<\mathbf{C}_{\mathrm{CM}}$ is always true under this adjustment. 


\subsection{Cost modeling uses}

Industry values cost reduction, product performance, and lead time, as these factors play key roles in sustainable manufacturing. AM meets all of these criteria, and through benchmarking, the advantages of AM are clearly outlined: AM can design and prototype parts within a short lead time, and the advancement of technologies and processes is more agile and faster than that observed for CM. Based on the illustration in this study, reducing AM's cost through new technologies to a lower cost than the convergence of the CM cost value is feasible. Through the business model, AM can achieve speed and scale, as well as cost savings, and stay in a competitive or superior position over CM by using this cost model supported by the establishment of a collaborative pattern. The strategy is described as follows:

The AM collaboration work may start with a focus on highly complex plastic components, which can help AM save materials and costs. Meanwhile, benchmarking against a high BTF ratio, as well as high tooling and machining products of $\mathrm{CM}$ can differentiate the advantages of AM in cost saving and to streamline the focus of AM. The steady relationships among LEs, SMEs, and HBMs and the mutual benefit can save the sales' margin and drop the cost to a very lower level than that of existing supply chains.

Machines and materials contribute $60 \%$ and $30 \%$, respectively, to AM cost. The material cost is expected to be lower than that of existing retailers. One reason for this is that instead of the supply chain, AM materials are fabricated by the local manufacturer, and the other reason is the AM community. Meanwhile, considering AM in short-term design-to-manufacturing and fast time-tomarket can also be a value proposition. Fully utilizing AM characteristics in product innovation based on AM flexibility can engage designers in redesigning products to expand the product scope and features, reduce material cost, and increase durability. In addition, collaborative patterns are proposed to consolidate the capacity of multientities' capacity and align their individual motivation and tendency into a robust workforce [23].

\section{BTF effectiveness}

BTF is the terminology commonly used in the aerospace industry to cite the ratio of the input weight of raw materials to the output weight of the product parts. As AM stays within an approximately 1:1 BTF ratio, the applications usually focus on those higher BTF ratios of $\mathrm{CM}$ and light weight of complex parts in which AM's advantages can be fully exploited [24]. BTF is generally used in metallic materials, though it can be used in plastics [25]. As CM is a subtractive technology, in a typical case, such as CNC, the BTF ratio ranges from 6:1 to 25:1 [26], and the averaged ratio is $11: 1$, depending on the materials and applications. Pereira [27] indicated that the higher the complexity or customization required, the better suited is $\mathrm{AM}$, which can also reduce energy consumption and $\mathrm{CO}_{2}$ emission. This effect is crucial, particularly for the parts that are used in aerospace, automobile, or any other lightweight applications [28]. In Northwest Airlines' case, the generic bracket, formed using 3D printers, saved over $10 \mathrm{~kg}$

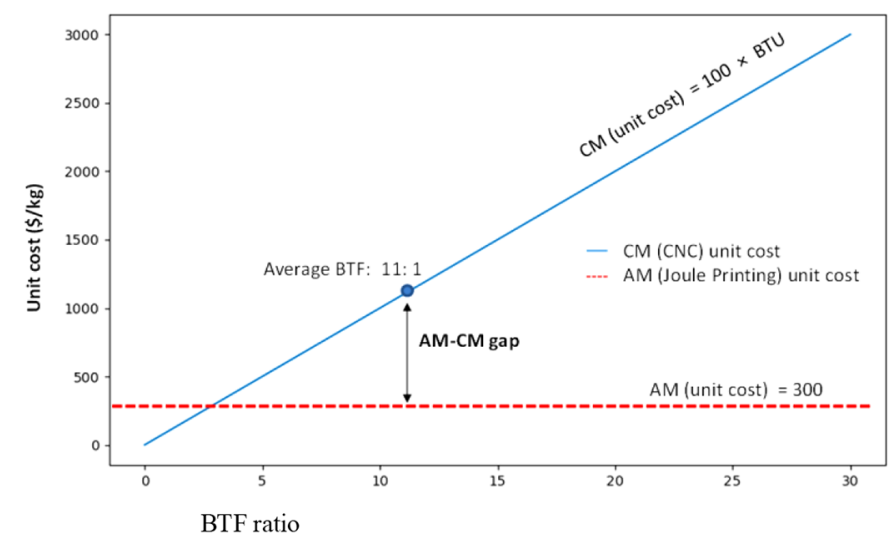

Fig. 4. AM-CM benchmark in Ti-64 case.

of weight, and the aircraft was expected to achieve cost savings of $\$ 440,000$ per year [29].

The Boeing Company evaluates a manufacturing method based on three key factors: cost, part performance, and lead time [30]. AM meets all three criteria, as its design and prototyping are fast, the lead time is short, and the cost can be reduced by using different technologies. Furthermore, with the advancement of technologies such as joule printing [31], AM can meet speed and scaling requirements. Figure 4 demonstrates how AM achieves better cost modeling by comparing the BTF ratio with the unit cost. The $x$-axis denotes the BTF ratio and the $\mathrm{y}$-axis denotes unit cost. The AM materials cost $\$ 300 / \mathrm{kg}: \mathrm{y}=300$. CNC comes with a lower material cost, $\$ 100 / \mathrm{kg}$, and stays with a simple equation: $\mathrm{y}=100 \mathrm{x}$. AM and $\mathrm{CM}$ costs intersect at $\mathrm{BTF}=3$. In the Ti-64 case, the BTF ratio is $17: 1$, and AM saves cost for $\$ 1400 / \mathrm{kg}$ compared to the CNC method of CM. Basically, BTF and CNC cases are used in alloy and seldom used in AM plastics manufacturing due to materials type and cost issues. Hence, BTF and CNC of $\mathrm{CM}$ method are for references only with no implication that these are the advantages of AM over CM in plastics manufacturing.

\subsection{AM weakness and limitations}

Compared to CM, AM is in its infancy, and standardization can be critical to supporting AM in becoming industry mainstream. Standardization starts with the materials' full lifecycle in terms of recycling, design, manufacturing, and quality assurance.

To establish a foundation to make the products more reproducible and reliable, test standards need to be in place [32]. According to ASTM, these standards cover materials, terminology, process, applications, design, and test methods. The standards define terminology, measure the performance of different production processes, ensure the quality of the end products and specify procedures for the calibration of AM machines [33].

1) A lack of industry standards can lead to a mismatch of mechanical properties' requirements and mislead energy calculation in the manufacturing process. The industry 
Table 8. Roles in collaborative pattern.

\begin{tabular}{ll}
\hline Role & Activities and responsibility \\
\hline LE & Companies that manage recycled and AM materials nearby materials recycling facility \\
SME & Companies that manage recycled materials, AM materials, and HBM services \\
HBM & Registered HBMs that deal with AM printing and products \\
\hline
\end{tabular}

standard means optimized alignments between technologies (i.e., FDM and injection molding), plastic types (i.e., PET and PLA), forms of materials (i.e., filament, powder, and their density), and applications (i.e., product's form and mechanical properties). As this is crucial to the ultimate application of AM and CM, this study will extend a separate investigation into application standards.

In addition, the form of feedstock (i.e., filament and powder) can depend on energy consumption. For instance, the filament for FDM is stable, but the calibration powder produced by recycled materials can consume more energy than the filament.

2) Prior to introducing the business model, this paper introduces the CRM model to produce a generic formula for the optimization method between recycling facilities and transportation distance calculation. It covers different technologies but might not cover the complete details of the process steps, such as chemical recycling or supply chain management, which shall be amended in a separate investigation and enlarge the scope of the cost model to the recycling method areas.

3) The energy consumption of AM and CM varies depending on the manufacturing technology and plastic type used. The investigation conducted prior to this study concluded that CM and AM consume similar energy levels per unit weight of the source material. However, the CM method, such as CNC, can consume higher energy (per unit weight of products) than the AM method if the BTF phenomenon is considered.

Energy consumption can be part of a cost model; however, due to ambiguity of the mapping between energy consumption and associated technologies, and the lack of relevant literature, it is a limitation of cost modeling, and a separate investigation is required to make the cost model a complete solution.

4) Scaling has been a bottleneck of AM in accelerating exponential growth in manufacturing, and this issue limits AM to a small scale, high complexity, and small size of parts, particularly in prototyping. To address the scaling issue in AM, AM technologies need to speed up innovations in automation and multi-tasking functions, such as multiple print heads or robots, to enhance AM's scaling capability.

5) Compared to CM, AM does not achieve a notable advantage in terms of material properties. The AM process can degrade polymer quality due to chain scission when water is included and acidic impurities are

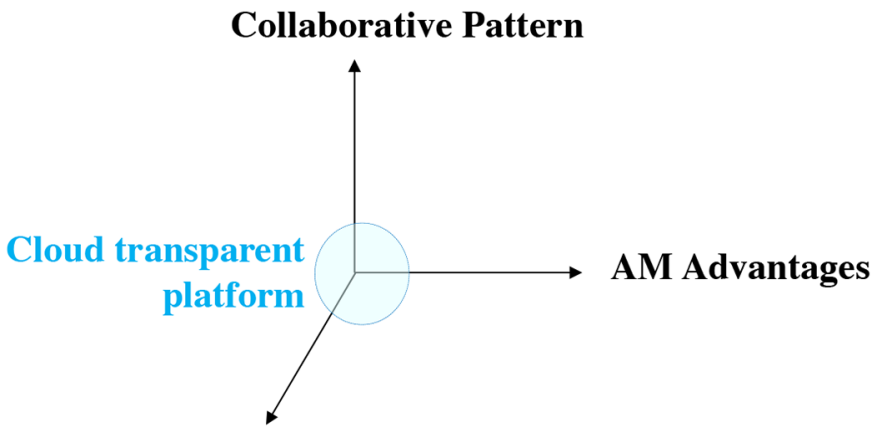

AM Applications

Fig. 5. Alignment among advantages, applications, and collaboration.

heated during the process. This inclusion can produce toxic substances from chlorine-containing polymers, which can affect public health. HBM/AM workers need open space and ventilation equipment to eliminate toxicity.

To consider the cost advantage of AM in production scaling resolution, this paper proposes a collaborative pattern through multiple roles (collaboration of LEs, SMEs, and HBMs). The collaborative pattern supports local recycling and manufacturing and reduces the supply chain, which can further reduce cost, transportation, and $\mathrm{CO}_{2}$ emission to address the demerits of AM.

\section{Collaborative pattern for localization}

To resolve AM issues related to volume limitation, slow manufacturing process, and product size, a collaborative pattern is proposed to support the business model to form a concrete AM-based society, to consolidate their individual strength, and to cumulate small but massive HBM into a robust workforce in the AM industry.

In this collaborative pattern, a 3D framework is proposed. As indicated in Figure 5, the alignment of AM's advantages to the appropriate applications and collaborative pattern through a fully transparent cloud platform is critical. Under this alignment, a concrete collaborative pattern can be built [34].

As indicated in Table 8, HBMs, SMEs, and LEs perform multiple roles in AM transformation. The collaboration of entities from an AM-based society enables the localization of the end-to-end process in the most dynamic manner. It covers material recycling, supply chains, and AM manufacturing. 


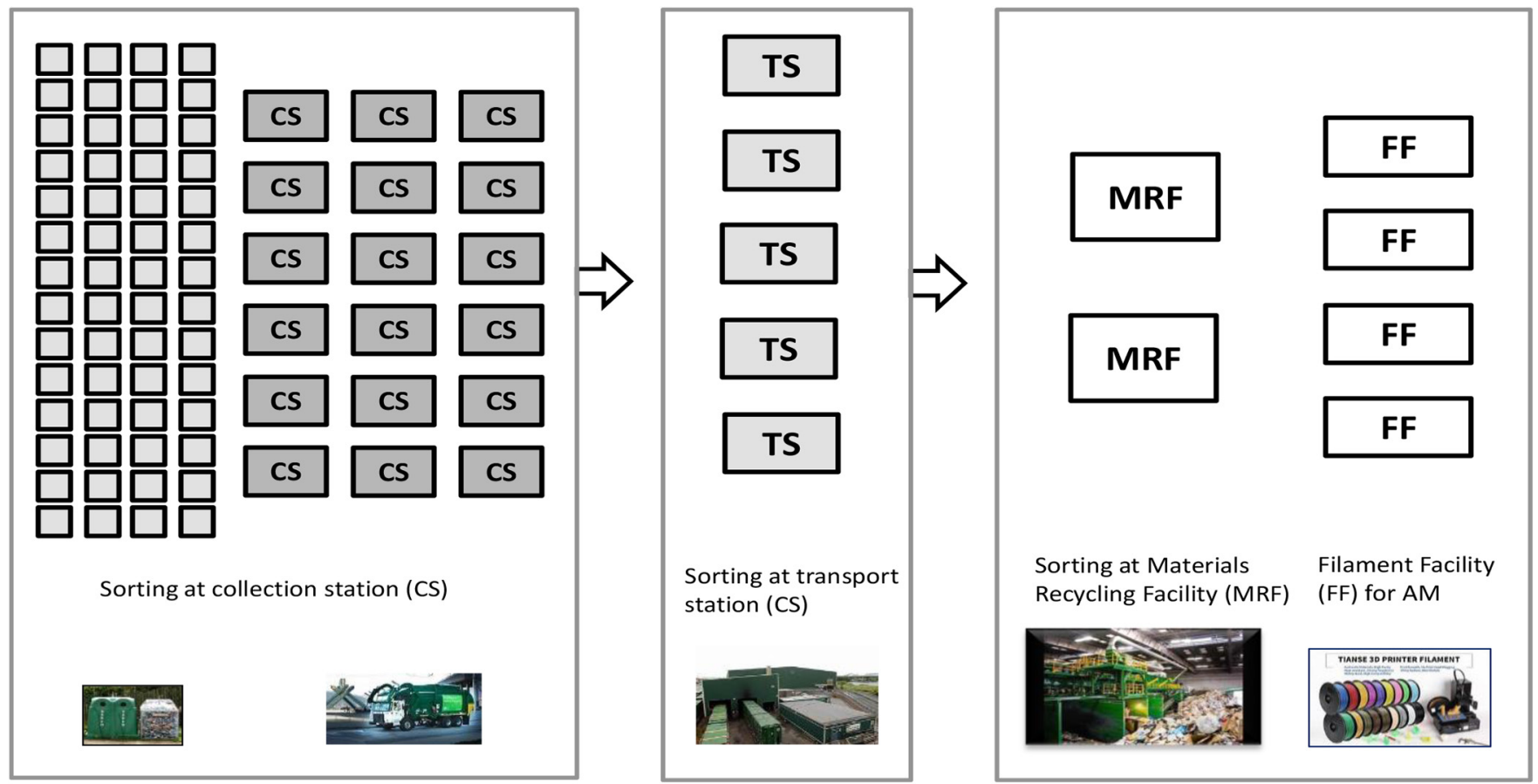

Fig. 6. Process topology of recycling and AM manufacturing.

\section{Case studies of collaborative patterns}

The Wabtec Corporation is a case study presented for multi-role collaboration. The company collaborated with Hewlett-Packard and launched an AM Center (Wabtec India AM Center), which focused on accelerating the design and production of integrated 3D-printed components in India. Wabttec-HP offers end-to-end solutions, consulting, and support to micro, small, and medium enterprises (MSMEs) and their individual customers, which is a typical example of collaboration among machine/material provider, SME, and HBM end-users [35].

\section{Roadmap of collaboration}

Task identification is expected to guide the collaboration strategy by taking a step-by-step approach to streamline the transformation. Meanwhile, rural inhabitants are encouraged to join the collaboration to establish a mutually beneficial AM-based society as strong urban rural partnerships create a resilient society [36]. In this roadmap, there are four stages of transformation and three types of roles for collaboration, which are defined as follows.

As illustrated in Figure 6, the key factors on which AM collaboration leverages are MRF recycling, AM source materials, AM technologies, services, 3D printing, AM standardization, Cloud platform, and AM product innovation.

To achieve ultimate coverage of collaboration, no entity should limit its role. For instance, an LE or SME can be a provider of AM printers, materials, or MRF facilitators, or a $100 \%$ pure manufacturer. An LE is mainly responsible for MRF and AM source materials; an SME covers AM printers, materials, and AM standards and is expected to promote HBM. HBM is randomly distributed in its AMbased society within a $100 \mathrm{~km}$ radius and can increase family income through a standardized process; consequently, they support SMEs and LEs in resolving AM volume issues. The topology of the collaborative domain is illustrated in Figure 6, where the collaboration focuses on MRF, filament facility (FF), and downstream distribution.

The four stages are outlined in Table 9 ;

A roadmap is necessary to streamline the AM transformation, minimize risk, and to establish a concrete foundation for the AM industry. Figure 7 demonstrates the mapping matrix among the three roles, key activities, and four stages.

\section{AM challenges}

Given that AM can be a future trend with tremendous advantages, potential or existing AM challenges cannot be ignored. AM encounters two major issues that need to be resolved before mass production begins.

1) AM is not ready for high-volume and large parts production at this stage, which limits it to small batch production. Compared to $\mathrm{CM}, \mathrm{AM}$ is a better fit for small-to-medium volumes of small parts and highquality products in the initial stage.

To address this issue, massive HBM workers can take advantage of AM's low entry cost, less space, and easy setup to start individual businesses right away. By cumulating a small but massive HBM, the volume issue of AM can be overcome, compatible with $\mathrm{CM}$ produc- 
Table 9. Key activities in 4 stages.

\begin{tabular}{|c|c|}
\hline Stage & Activities \\
\hline Stage 2 & $\begin{array}{l}\text { Distribution: a distribution topology of AM community at } 100 \mathrm{~km} \text { MRF surrounding is setup } \\
\text { HBM starts to setup their printers and filaments based on their products selection. } \\
\text { Government and authorities are suggested to launch loan or minimum financial support to } \\
\text { HBM to setup HBM without any financial concern. HBM starts to receive minimum training } \\
\text { in AM printings. } \\
\text { SME analyzes company's key strength and decide the AM business strategy and start to add } \\
\text { or open new AM business. SME takes advantage of scale economics offered by AM as a } \\
\text { potential enabler of supply chain transformation for the specific products. }\end{array}$ \\
\hline
\end{tabular}

tion. Furthermore, the multitasking approach at Bennett Plastics is a case study explaining that one worker is sufficient to handle multiple 3D-printing machines to increase productivity [38]. In addition, adding multiple print heads can aid in increasing the speed of AM [39]. Once the scaling issue is resolved, the speed issue can be easily resolved, and the size issue can be resolved onsite through multipart assembly.

2) Mechanical recycling is one of the most economical methods of recycling, particularly for single-polymer and less-purity plastic materials. Mechanical recycling is limited to single polymers, such as PE, PS, and PP, and quality degradation can be caused by heat and energy supply during recycling, which results in photo-oxidation and internal stresses to the materials produced by the AM process [40].

Eventually, the material strength is degraded [41]. To prevent quality degradation caused by the recycling process, this paper proposes the installation of dehumidification equipment to eliminate moisture, diluting oxygen by nitrogen injection, or the use of a vacuum to eliminate oxidization to prevent the degradation of material properties.

3) AM production relies on a unique vision of multientities to establish standards, common practices, and sharable values; however, in the current situation, this is a challenge to AM, and communication can be a major drawback. 


\begin{tabular}{l|l|l}
\multicolumn{1}{c|}{ Role } & \multicolumn{1}{c}{ Key Responsibility } & \multicolumn{1}{c}{ Deliverables } \\
\hline LE (large enterprise) & Manage MRF, AM materials & AM source and refining materials \\
\hline SME (small to medium enterprise) & AM facilities, standard and service & AM printers, materials delivery and services \\
\hline HB (home business) & AM printing and products innovation & AM Products
\end{tabular}

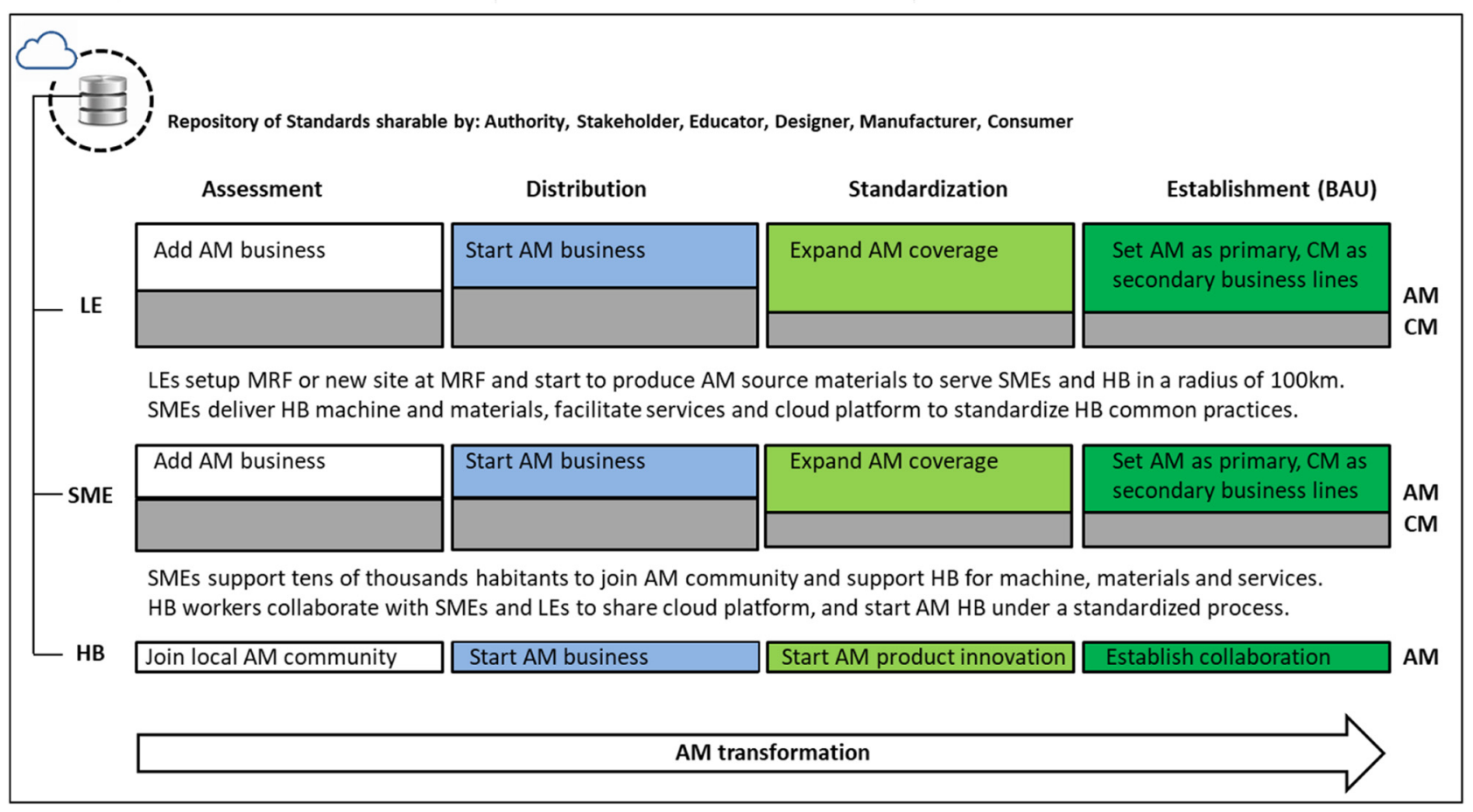

Fig. 7. Roadmap of collaborative pattern -3 roles in 4 stages.

To address this issue, this study also encourages a wider scope of multi-entities, such as academia, enterprises, government, manufacturers, consumers, and AM designers, to establish a common platform and forum to share information in full transparency. Traceability and tracking are critical mechanisms to guide AM technologies into the mainstream through multi-entity communications and standardization processes.

\section{Conclusion}

AM is an emerging technology whose advantages are foreseen by industry; however, many companies still hesitate to use AM, particularly traditional enterprises that do not involve risk-taking as part of their business plan. These companies understand the advantages of AM over CM, such as shorter lead time, better quality, easy prototyping of high complexity, flexible design, and human-centric linkage to state-of-the-art technologies; however, cost savings through AM can be a bottleneck.

The business model, which is the focus of this study, is crucial to streamline the roadmap between technologies and production. Stakeholders require evidence that AM can be a cost-effective method without much concern in production, and decision makers require indicators of cost savings in either AM or CM under the constraints of technology, volume, and lead time.

This paper proposes a "divide-and-conquer" cost modeling approach to investigate the bottleneck. CM production needs a high volume to share initial tooling costs before cost savings can be achieved; however, the cost of AM is volume-independent. In a more expensive AM technology, the unit costs of AM and CM intersect at a certain point of volume, at which $\mathrm{CM}$ can save cost if the batch volume crosses the intersection point. However, CM convergence, the limitation in which a high batch volume can be beneficial to cost saving, can lose its advantage if any AM technology lesser than CM convergence, such as FDM, is proposed. Decision makers can easily input conditions and foresee the cost-saving option; in contrast, they can preset the range of cost estimation and select the best conditions for the ultimate values of parts or products.

In addition, an AM collaborative pattern is proposed to address speed, scale, and size issues. In this AM-based society, collaborative patterns are expected to connect LEs, SMEs, and HBM to realize localization and distributed manufacturing. A collaborative pattern is not only expected 
to handle the AM bottleneck in these gaps but is also a critical approach within the business model that minimizes the dependency of AM on SCM and can achieve additional cost savings in logistics, warehousing, and transportation. Consequently, it reduces the energy and $\mathrm{CO}_{2}$ emission of plastic manufacturing toward sustainability.

\section{References}

1. S.M. El-Haggar, M. Salah, Sustainable Industrial Design and Waste Management (Elsevier Academic Press, 2007)

2. UNDP Converting Waste Plastics into a Resource", Compendium of Technologies, United Nations Environment Programme 2009;

3. A.N. Nambiar, Challenges in sustainable manufacturing, in International Conference on Industrial Engineering and Operations Management, Bangladesh, India, January 9-10, 2010 (2010)

4. M. Despeisse, M. Baumers, P. Brown, F. Charnley, S.J. Forda, A. Garmulewicz, S. Knowles, T.H.W. Minshall, L. Mortara, F. P. Reed-Tsochas, J. Rowleyh, Unlocking value for a circular economy through 3D printing: a research agenda, Technolog. Forecast. Social Change 115 (2017) 75-84

5. A.M. Tofail, E.P. Koumoulos, A. Bandyopadhyay, S. Bose, L. O'Donoghue, C. Charitidis, Additive manufacturing: scientific and technological challenges, market uptake and opportunities, Mater. Today 21 (2018) 22-37

6. R. Huang, M. Riddle, D. Graziano, J. Warren, S. Das, S. Nimbalkar, J. Cresko, E. Masaneta, Energy and emissions saving potential of additive manufacturing: the case of lightweight aircraft components, J. Clean. Prod. 135 (2016) 1559-1570

7. J. Lee, J. Chee, K. Chua, Fundamentals and applications of 3D printing for novel materials, Appl. Mater. Today 7 (2017) 120-133

8. GE Additive, Speed and Scale in the Spinal Sector (2020) accessed at: http://additivemanufacturing.com/2020/11/ 09/ge-additive-speed-and-scale-in-the-spinal-sector/

9. S. Muthu, M. Savalani, Handbook of Sustainability in Additive Manufacturing (Springer, Singapore, 2016), Vol. 1

10. K. Wong, A. Hernandez, A review of additive manufacturing, ISRN Mech. Eng. 2012 (2012)

11. J. Butt, Exploring the interrelationship between additive manufacturing and industry 4.0", Designs 4 (2020) 13

12. T.D. NgoaAlirez, A. Kashani, G. Imbalzanoa, K.T.Q. Nguyen, D. Hui, Additive manufacturing (3D printing): a review of materials, methods, applications and challenges, Compos. B: Eng. 143 (2018) 172-196

13. S. Ford, M. Despeisse, Additive manufacturing and sustainability: an exploratory study of the advantages and challenges, J. Cleaner Prod. 137 (2016) 1573-1587

14. D.S. Thomas, S.W. Gilbert, Cost Effectiveness of Additive Manufacturing: A Literature Review and Discussion, US National Institute of Standards and Technology (NIST Special Publication 1176) (2014) accessed at: http://dx.doi. org/10.6028/NIST.SP.1176

15. J.W.N. Smith, G. Kerrison, Benchmarking of decisionsupport tools used for tiered sustainable remediation appraisal, Water Air Soil Pollution 224 (2013)

16. E.A. Posner, K. Spier, A. Vermeule, Divid and Conquer, Harvard Law School (2009) accessed at: Harvard paper
17. H. Lockett, Additive Manufacturing - helping to reduce waste in aircraft production, Design research (2019) accessed at: http://www.open.ac.uk/

18. S. Vyavahare, S. Teraiya, D. Panghal, S. Kumar, Fused deposition modelling: a review, Rapid Prototyp. J. 26 (2020) 176-201

19. M. Cotteleer, 3D opportunity for production: Additive manufacturing makes its (business) case, Deloitte Rev. (2014) assessable at: https://www2.deloitte.com/us/en/ insights/deloitte-review/issue-15/additive-manufacturingbusiness-case.html

20. Shapeways Will Additive Manufacturing Replace Conventional Manufacturing? 2021; https://www.shapeways. com

21. T.W. Simpson, The Value of Design for Additive Manufacturing (DFAM), Addit. Manufactur. (2020) accessed at: https:// www.additivemanufacturing.media/articles/the-value-of-de sign-for-additive-manufacturing-dfam

22. T. Simpson, Additive manufacturing via material extrusion, Additive Manufacturing (2019) assessed at: https://www. additivemanufacturing.media/articles/additive-manufactur ing-with-material-extrusion

23. V. Ghomashchi, Building sustainability through collaborative planning, Int. J. Sustain. Dev. Plan. 7 (2012) 14-25

24. Z. Lin, K. Song, X. Yu, A review on wire and arc additive manufacturing of titanium alloy, J. Manufactur. Process. 70 (2021) 24-45

25. Reportlinker Global Aerospace Additive Manufacturing Industry 2020; https://www.prnewswire.com

26. E. Holm, V. Valencia, A. Thal, J. Freels, A. Badiru, Materials Testing and Cost Modeling for Composite Parts Through Additive Manufacturing (Air Force Institute of Technology, 2011) accessed at: efaidnbmnnnibpcajpcgl

27. T. Pereira, J. Kennedy, J. Potgieter, A comparison of traditional manufacturing vs additive manufacturing, the best method for the job, 14th Global Congress on Manufacturing and Management, Proc. Manufactur. 30 (2018) 11-18

28. Spartacus3D How can Additive Manufacturing help the Aerospace Sector? 2014; https://www.farinia.com/blog

29. S. Fried, Shaping the future of additive manufacturing and 3D printed electronics, Nano Dimension Blog (2019) accessed at: https://www.nano-di.com/

30. K. Sertoglu, Boeing takes to the sky with Chinook's first 3D Printed flight-critical par (2021) accessed at: https:// 3dprintingindustry

31. Digital Alloys Comparison of Additive Manufacturing \& CNC Machining 2019; https://www.digitalalloys.com/blog/ comparison-additive-manufacturing-cnc-machining

32. J. Dizona, Mechanical characterization of 3D-printed polymers, Addit. Manufactur. 20 (2018) 44-67

33. ASTM International Additive Manufacturing Technology Standards 2021; https://www.astm.org/Standards/addi tive-manufacturing-technology-standards.html

34. E. Rauch, S. Seidenstricker, P. Dallasega, P. Hämmer, Collaborative cloud manufacturing: design of business model innovations enabled by cyberphysical systems in distributed manufacturing systems, J. Eng. 2016 (2016) Article ID 1308639

35. A. Chandavarkar, Wabtec inaugurates Additive Manufacturing Center in Bengaluru, AM Chronicle (2020). Accessed at: https://www.amchronicle.com/news/wabtecinaugurates-additive-manufacturing-center-in-bengaluru/ 
36. R. Shaw, Urban Rural Partnership: A Win-Win Solution Towards Resilient Growth, URBANET (2019) accessed at: https://www.urbanet.info/urban-rural-part nership/

37. W. Heising, Additive Manufacturing Needs a Business Ecosystem (Boston Consulting Group, 2020) accessed at: https://www.bcg.com/publications/2020/additivemanufacturing-needs-to-adopt-a-managed-business-ecosys tem

38. K. Hanna, From prototypes to production, manufacturers harness evolving 3-D technology, Addit. Manufactur. (2021) assessed at: https://www.plasticsmachinerymanufacturing. com

39. M. Attaran, The rise of 3-D printing: the advantages of additive manufacturing over traditional manufacturing, Bus. Horizons 60 (2017) 67-688

40. R. Pinsky, P. Sabharwall, A. Gaffney, Energy input and process flow for plastics recycling, Int. J. Eng. Sci. Res. Technol. 8 (2019) 230-243

41. S. Al-Salem, P. Lettieri, J. Baeyens, Recycling and recovery routes of plastic solid waste (PSW): a review, Waste Manag. 29 (2009) 2625-2643

Cite this article as: Haishang $\mathrm{Wu}$, Business model and methods of evaluation in sustainable manufacturing, Manufacturing Rev. 8, $28(2021)$ 